\title{
Hypothyroidism in polycystic ovarian syndrome: a comparative study of clinical characteristics, metabolic and hormonal parameters in euthyroid and hypothyroid polycystic ovarian syndrome women
}

\section{Ramanand $\mathrm{SJ}^{1}$, Raparti GT ${ }^{2}$, Halasawadekar $\mathrm{NR}^{1}$, Ramanand $\mathrm{JB}^{3}$, Kumbhar AV ${ }^{1}$, Shah $\mathrm{RD}^{1}$}

\author{
${ }^{1}$ Department of Pharmacology, Government Medical College, Sangli, Maharashtra, India \\ ${ }^{2}$ Department of Pharmacology, Ashwini Rural Medical College and Hospital, Solapur, Maharashtra, India \\ ${ }^{3}$ Department of Pharmacology, R.C.S.M. Government Medical College, Kolhapur, Maharashtra, India
}

Received: 22 July 2016

Accepted: 16 August 2016

\author{
*Correspondence: \\ Dr. Raparti GT, \\ E-mail: girishraparti@yahoo.in
}

Copyright: () the author(s), publisher and licensee Medip Academy. This is an open-access article distributed under the terms of the Creative Commons Attribution Non-Commercial License, which permits unrestricted non-commercial use, distribution, and reproduction in any medium, provided the original work is properly cited.

\section{ABSTRACT}

Background: This study was conducted to examine influence of hypothyroidism on pathophysiology and features of PCOS with respect to clinical characteristics of polycystic ovarian syndrome (PCOS), hormonal and metabolic profile.

Methods: 102 euthyroid PCOS and 18 hypothyroid PCOS women were included in this cross-sectional study after considering inclusion and exclusion criteria. The study subjects were assessed for various signs and symptoms like recent weight gain, obesity, abnormal hair growth, hirsutism, hair loss, acne, acanthosis nigricans and infertility. Various hormonal and metabolic parameters were evaluated viz. Luteinizing hormone, Follicle stimulating hormone, LH:FSH ratio, testosterone, prolactin, dehydroepiandrosterone, fasting insulin and fasting blood glucose. BMI and HOMA values were calculated.

Results: Association of hirsutism, excessive hair growth, hair loss, acanthosis nigricans, acne, infertility was not significant between the two groups. Majority of patients in both the groups were overweight/obese. BMI and number of patients complaining weight gain was significantly more in hypothyroid PCOS women. While no statistical difference in LH, FSH, LH:FSH ratio, prolactin, and testosterone levels was found, serum DHEA level was significantly less in hypothyroid PCOS group. No statistical difference in fasting blood glucose and insulin levels was found between the two groups. Though both the groups show insulin resistance, HOMA values were significantly more in hypothyroid PCOS women.

Conclusions: Presence of hypothyroidism significantly increased severity of insulin resistance as well as obesity in PCOS. This could have adverse metabolic consequences in them. Concurrent occurrence of both these disorder could also possibly affect other features of the PCOS viz. hair loss and infertility.

Keywords: PCOS, Hypothyroidism, Obesity, BMI, HOMA, DHEA, Infertility

\section{INTRODUCTION}

Coexistence of hypothyroidism and polycystic ovary syndrome (PCOS) has been documented. ${ }^{1}$ Though the underlying causes of PCOS and hypothyroidism are different; irregular menstrual cycles, infertility and obesity are the common features of both these disorders. The overlapping features of PCOS and hypothyroidism can lead to the failure of clinical diagnosis of one of the disorders. Both these disorders influence the reproductive and metabolic functions adversely.

Alterations in hormonal and metabolic parameters can occur in PCOS as well as hypothyroid women. Insulin resistance is a common feature in PCOS women. There is an association between thyroid function and insulin 
sensitivity. How these disorders influence each other or the relation between these two disorders, if any, is less understood. Hirsutism, baldness and acanthosis nigricans are the other clinical features of PCOS. All women of PCOS do not show all the clinical characteristics or derangement in biochemical parameters to the same extent. A possibility of hypothyroid state, influencing severity of PCOS adversely, may exist. The present study was conducted to throw a light on these aspects. PCOS patients with euthyroidism and hypothyroidism were compared with respect to clinical characteristics of PCOS, hormonal and metabolic profile.

\section{METHODS}

This was a cross-sectional study conducted in PCOS women. The cohort was similar to previously published research article. ${ }^{2}$ One hundred twenty newly diagnosed post pubertal PCOS women of reproductive age consulting endocrinology hospital were enrolled in the present study as per inclusion and exclusion criteria after taking written informed consent. The diagnosis of PCOS was fulfilled as per Rotterdam criteria. ${ }^{3}$ Presence of at least two criteria from clinical, hormonal, abdominal USG category, were considered diagnostic of PCOS. Patients having any other major systemic illness including congenital adrenal hyperplasia, hyperprolactinemia, acromegaly, functional hypothalamic amenorrhea were excluded.

Detailed menstrual history, marital status, parity were recorded in both the groups. All the study subjects were assessed for various signs and symptoms like recent weight gain, obesity, abnormal excessive hair growth, hirsutism, hair loss/ baldness, acne and acanthosis nigricans. Infertility was assessed only in married women. Depending on feasibility, enrolled patients were investigated for various hormonal and metabolic parameters viz. serum levels of Luteinizing hormone (LH), Follicle stimulating hormone (FSH), LH:FSH ratio, testosterone, prolactin (PRL), dehydroepiandrosterone (DHEA), fasting insulin and fasting blood glucose (FBG); after taking overnight fasting blood sample. In vitro quantitative determination of hormones was carried by electrochemiluminescence immunoassay method (Cobas e 411). Blood glucose was measured on auto analyser (Microlab 300) using oxidase method.

Body mass index (BMI) of all the subjects was calculated. Cut-off of BMI as standard consensus statement for Indian population was considered. ${ }^{4}$ BMI value more than $23 \mathrm{Kg} / \mathrm{m}^{2}$ was considered as overweight. Homeostatic model assessment insulin resistance index (HOMA-IR) was calculated as marker of insulin resistance. Value of HOMA >1.9 was considered as presence of insulin resistance.

Patients were observed for signs and symptoms of hypothyroidism and investigated for serum thyroidstimulating hormone (TSH) levels. Normal TSH level was considered as 0.5-4.5 mIU/L. Serum T3; T4 levels were estimated in patients showing raised TSH levels. Normal freeT3 and T4 levels were considered as 2.4-4.2 $\mathrm{pg} / \mathrm{ml}$ and $0.7-1.24 \mathrm{ng} / \mathrm{dl}$ respectively. Women with elevated levels of TSH with normal T3 and T4 levels and without signs and symptoms of hypothyroidism were considered as subclinical hypothyroid patients. Those showing signs and symptoms of hypothyroidism along with increased TSH and decreased free T3 and T4 levels considered as patient with overt hypothyroidism. PCOS women with subclinical and overt hypothyroidism were grouped as hypothyroid PCOS women. Other PCOS patients who did not show raised TSH levels were grouped as euthyroid PCOS women.

Out of 120 PCOS patients, 18 were having hypothyroidism and were put on treatment with levothyroxine. Among these 18 hypothyroid PCOS women, two PCOS women had subclinical hypothyroidism. Remaining 102 PCOS women were euthyroid. Thirty eight women in euthyroid and nine women in hypothyroid PCOS subgroup were married. The study was approved by institutional ethics committee. All the guidelines of Helsinki were followed. Data was analysed statistically using software (Graph pad prism version 6). All the data was tested for normality. Two groups were compared for presence of clinical characteristics of PCOS by either chi-square test or Fisher's exact test. Fisher's exact test was applied for small sample size of data. The quantitative data was presented as Mean \pm SD and compared by using either unpaired ' $t$ ' test for normally distributed data or Mann Whitney test for data not normally distributed. All hypothesis tests were two-sided and $\mathrm{p}$ value less than 0.05 were considered as significant.

\section{RESULTS}

Qualitative and quantitative data of 102 euthyroid PCOS women was compared with 18 hypothyroid PCOS women. The mean age in years was 21.74 4.31 (range 14 to 33 ) in euthyroid PCOS and 23.83 6.04 (range 15 to 35 ) in hypothyroid PCOS patients. The mean age was statistically comparable $(\mathrm{p}=0.077)$. The mean weight (in $\mathrm{kg}$ ) of euthyroid and hypothyroid PCOS women was $63.74 \pm 16.67$ and $70.61 \pm 16.58$ respectively. This difference was not significant $(\mathrm{p}=0.082)$. The mean BMI of euthyroid (after deleting outlier value of one patient weighing $128 \mathrm{kgs}$ ) and hypothyroid PCOS women was $26.66 \pm 5.75$ and $29.79 \pm 6.23$ respectively. This difference was found statistically significant $(\mathrm{p}=0.037)$.

Clinical characteristics between two groups were compared (Table 1). Association of hirsutism, excessive hair growth, hairloss/baldness, acanthosis nigricans, acne, infertility was compared between the two groups and found non-significant. Infertility was assessed in married women only. Number of patients complaining weight gain was significantly more in hypothyroid PCOS women $(\mathrm{p}=0.021)$. Various hormonal parameters were compared 
between the two groups in table 2. It was seen that there was no statistical difference in LH, FSH, LH:FSH ratio, prolactin, and testosterone levels between the two groups. While serum DHEA level was significantly less in hypothyroid PCOS women than euthyroid PCOS group. Table 3 shows comparison of metabolic parameters between euthyroid and hypothyroid groups of PCOS women. When glycemic parameters were compared, it was seen that differences in fasting blood glucose (FBG) and insulin between the two subgroups was not significant. HOMA of hypothyroid PCOS group was significantly more than euthyroid PCOS group of women.

Table 1: Comparison of clinical characteristics of euthyroid and hypothyroid PCOS women.

\begin{tabular}{|c|c|c|c|c|}
\hline Clinical characteristics & Present/ Absent & Euthyroid PCOS n = $102(\%)$ & Hypothyroid PCOS n=18 (\%) & p value \\
\hline \multirow{2}{*}{ c/o weight gain } & Present & $23(22.55)$ & $9(50)$ & \multirow{2}{*}{$0.021 \# *$} \\
\hline & Absent & $79(77.45)$ & $9(50)$ & \\
\hline \multirow{2}{*}{$\begin{array}{l}\text { Overweight and obese } \\
\text { (BMI>23) }\end{array}$} & Present & $73(71.57)$ & $16(88.89)$ & \multirow{2}{*}{$0.15 \#$} \\
\hline & Absent & $29(28.43)$ & $2(11.11)$ & \\
\hline \multirow{2}{*}{ c/o excessive hair growth } & Present & $12(11.76)$ & $3(16.67)$ & \multirow{2}{*}{$0.69 \#$} \\
\hline & Absent & $90(88.24)$ & $15(83.33)$ & \\
\hline \multirow{2}{*}{ Hirsutism } & Present & $45(44.12)$ & $8(44.44)$ & \multirow{2}{*}{0.97} \\
\hline & Absent & $57(55.88)$ & $10(55.56)$ & \\
\hline \multirow{2}{*}{$\begin{array}{l}\text { Hair loss/ } \\
\text { Baldness }\end{array}$} & Present & $5(4.90)$ & $3(16.67)$ & \multirow{2}{*}{$0.098 \#$} \\
\hline & Absent & $97(95.10)$ & $15(83.33)$ & \\
\hline \multirow{2}{*}{ Acne } & Present & $22(21.57)$ & $2(11.11)$ & \multirow{2}{*}{$0.52 \#$} \\
\hline & Absent & $80(78.43)$ & $16(88.89)$ & \\
\hline \multirow{2}{*}{ Acanthosis nigricans } & Present & $43(42.16)$ & $10(55.56)$ & \multirow{2}{*}{0.29} \\
\hline & Absent & $59(57.84)$ & $8(44.44)$ & \\
\hline \multirow{2}{*}{ Infertility } & Present & $15(39.47)$ & $6(66.67)$ & \multirow{2}{*}{$0.26 \#$} \\
\hline & Absent & $23(60.53)$ & $3(33.33)$ & \\
\hline
\end{tabular}

\#- Fishers exact test, others chi-square test, only married women were considered for infertility.

Table 2: Comparison of hormonal parameters (mean \pm sd) between euthyroid and hypothyroid groups of PCOS women.

\begin{tabular}{|c|c|c|c|c|c|c|}
\hline & LH IU/L & FSH IU/L & LH/FSH ratio & Prolactin ng/dl & DHEA $\mu \mathrm{g} / \mathrm{ml}$ & Testosterone $\mathrm{ng} / \mathrm{ml}$ \\
\hline $\begin{array}{l}\text { Euthyroid } \\
\text { PCOS }\end{array}$ & $\begin{array}{l}12.54 \pm 5.87 \\
(102)\end{array}$ & $\begin{array}{l}5.70 \pm 1.80 \\
(102)\end{array}$ & $2.23 \pm 0.94(102)$ & $12.91 \pm 6.16(97)$ & $360.9 \pm 238.6(60)$ & $\begin{array}{l}1.21 \pm 3.00 \\
(44)\end{array}$ \\
\hline $\begin{array}{l}\text { Hypothyroid } \\
\text { PCOS }\end{array}$ & $\begin{array}{l}10.38 \pm 4.16 \\
(18)\end{array}$ & $\begin{array}{l}5.32 \pm 1.54 \\
(18)\end{array}$ & $2.05 \pm 0.89(18)$ & $17.90 \pm 14.19(15)$ & $215.5 \pm 106.7(8)$ & $\begin{array}{l}2.93 \pm 6.35 \\
(6)\end{array}$ \\
\hline p value & 0.13 & $0.45 \#$ & $0.42 \#$ & $0.26 \#$ & $0.044 \# *$ & $0.89 \#$ \\
\hline
\end{tabular}

\#-Mann Whitney; others t test; Numbers in bracket indicate sample size

Table 3: Comparison of metabolic parameters (mean \pm sd) between euthyroid and hypothyroid groups of PCOS women.

\begin{tabular}{|llll|}
\hline Euthyroid PCOS & FBG $(\mathrm{mg} / \mathrm{dll})$ & Insulin $(\mathrm{mU} / \mathrm{ml})$ & HOMA \\
\hline Hypothyroid PCOS & $89.44 \pm 12.95(39)$ & $14.52 \pm 9.55(28)$ & $3.16 \pm 1.87(28)$ \\
\hline p value & $90.42 \pm 15.60(11)$ & $24.47 \pm 17.10(9)$ & $5.18 \pm 3.54(9)$ \\
\hline
\end{tabular}

\#-Mann Whitney; others t test; Numbers in bracket indicate sample size

\section{DISCUSSION}

The polycystic ovary syndrome (PCOS) is one of the commonest endocrinological disorder affecting women in the reproductive age group. Some of the PCOS women show presence of hypothyroidism. The latter condition could affect pathophysiology and features of PCOS. The present study was aimed to compare clinical presentation of PCOS and biochemical parameters in euthyroid and hypothyroid PCOS women, which could throw a light on influence of hypothyroidism in PCOS.

Stein and Leventhal have recognized obesity as a common feature of PCOS. ${ }^{5}$ Hypothyroidism is also 
commonly associated with obesity. Even slightly elevated serum TSH levels are associated with an increase in the occurrence of obesity. ${ }^{6}$ In the present study, majority of patients in both the groups were overweight/obese (Table 1). Though the difference between numbers of overweight/obese women in both the groups was statistically insignificant, BMI of hypothyroid PCOS women was significantly more than the euthyroid PCOS group. Therefore, presence of hypothyroidism could exaggerate the severity of obesity in PCOS women, which could render them to its adverse consequences. Furthermore, the number of PCOS women complaining of weight gain was significantly higher in hypothyroid as compared to euthyroid group of PCOS women. The reason could be that the substantial gain in body weight within a short span of time in hypothyroid PCOS women was noticed easily.

Hirsutism is one of the characteristic features of PCOS. In the present study, percentage of women complaining of abnormal excessive hair growth and showing presence of hirsutism in euthyroid PCOS and hypothyroid PCOS groups was comparable (Table 1). PCOS women may also present with complaint of loss of hair. Futterweit W et al suggested that patients with diffuse alopecia may demonstrate hyperandrogenism, even in the absence of hirsutism, oligomenorrhea, or amenorrhea. The most common endocrine disorder in this series of patients with diffuse alopecia was polycystic ovarian disease. ${ }^{7}$ Diffuse hair loss is sometimes the presenting symptom of hypothyroidism. $^{8}$ It is well-known that thyroid hormone is essential for the development and maintenance of the hair follicle. Sterry W et al in their study reported that trichograms from the parietal and occipital areas showed increased dysplastic and broken hairs suggesting that alopecia in thyroid disease is not caused by changes within hair cycle, but probably by impaired hair quality. ${ }^{9}$ The present study indicates that hypothyroidism may enhance the occurrence of baldness in PCOS women as more percentage of hypothyroid women had hair loss (Table 1). Failure to reach the level of significance may be due to less number of hypothyroid PCOS women.

Acanthosis nigricans in PCOS is attributed to insulin resistance. Hypothyroidism can be added to the list of endocrine diseases associated with acanthosis nigricans. This is unlikely to be a direct effect of the hypothyroid state, but may instead be an indirect action mediated through obesity and subsequent insulin resistance. Insulin resistance and hyperinsulinemia seem to be commonly associated with (and perhaps causative of) the acanthosis nigricans that is seen in many endocrine diseases. ${ }^{10}$ All women showing presence of acanthosis nigricans, in both the study groups, were insulin resistant with increased HOMA values. All the patients showing acanthosis nigricans were obese (data not shown).

Infertility is common in both PCOS and hypothyroidism. Pfeifer SM et al have mentioned infertility as one of the long term sequelae of PCOS. ${ }^{11}$ Prevalence of hypothyroidism is $2-4 \%$ in women in the reproductive age group. Hypothyroidism can affect fertility due to anovulatory cycles, luteal phase defects, hyperprolactinemia, and sex hormone imbalance. ${ }^{12}$ In the present study, the percentage of infertile (married women) in hypothyroid PCOS group was greater than that in the euthyroid PCOS (Table 1). Failure to reach the level of significance may be because of less number of hypothyroid PCOS women. However, present study result suggests that hypothyroid state may accentuate infertility in PCOS women. Mean values of LH, FSH and LH/FSH ratio were found insignificantly less in hypothyroid PCOS women in this study. Mean values of serum prolactin and testosterone were greater in hypothyroid PCOS group, though not significant.

In the present study, mean DHEA was within reference range in both the groups. Hypothyroid PCOS women had lesser mean DHEA levels as compared to euthyroid PCOS women. The difference was statistically significant (Table 2). Tagawa $\mathrm{N}$ et al had reported decreased serum DHEA in hypothyroid patients. The authors have explained that lower DHEA in hypothyroidism could be because of decreased adrenal steroidogenesis induced by the low concentration of thyroid hormones. ${ }^{13}$ This could explain lower DHEA levels in hypothyroid PCOS women. Casson PR et al in a case series of treatment of infertile women have reported that DHEA appears to augment ovulation induction in poor responders. ${ }^{14}$ This could suggest possible association between decreased DHEA and infertility in hypothyroid patients.

Insulin resistance was observed in both euthyroid and hypothyroid PCOS groups of women in this study, suggested by high mean HOMA values. The severity of insulin resistance was more in hypothyroid PCOS group of women, as indicated by significantly higher HOMA values in them (Table 3). Dunaif et al found that women with PCOS are profoundly insulin resistant and are at an increased risk of development of type 2 diabetes mellitus. ${ }^{15}$ Erini $\mathrm{M}$ have reported presence of insulin resistance in hypothyroidism and subclinical hypothyroidism. ${ }^{16}$ Dittrich $\mathrm{R}$ et al have reported altered insulin resistance indices with higher fasting insulin concentration in women with PCOS and with TSH $\geq 2.5$ mIU/l. ${ }^{17}$ This increased severity of insulin resistance as well as obesity could have adverse metabolic consequences in hypothyroid PCOS women.

Drug like metformin which improves insulin sensitivity is used in PCOS. Isidro LM et al have reported that shortterm metformin administration is associated with a significant fall in TSH in obese, diabetic patients with primary hypothyroidism on thyroxine replacement therapy. ${ }^{18}$ These treatment modalities together implies beneficial role of metformin in hypothyroid PCOS women. The present study was a cross-sectional study carried out in relatively small number of patients. More extensive prospective studies including large number of patients to evaluate long term effects of various treatment 
modalities, like levothyroxine, metformin etc., on different clinical and biochemical parameters can be done. On the other hand, it is stated that optimal level of DHEA is needed in reproduction to maintain fertility. ${ }^{19}$ Therefore further studies assessing serum levels of DHEA in hypothyroid PCOS women after levothyroxine therapy may explore role of DHEA/supplements in the treatment of infertility.

To conclude, the present study suggests that coexistence of hypothyroidism significantly increase the severity of insulin resistance as well as obesity in patients with PCOS. This could have adverse metabolic consequences in them. Presence of hypothyroidism could possibly influence on other features of PCOS as well viz. hair loss and infertility.

\section{Funding: No funding sources}

Conflict of interest: None declared

Ethical approval: The study was approved by the Institutional Ethics Committee

\section{REFERENCES}

1. Sinha U, Sinharay K, Saha S, Longkumer TA, Baul $\mathrm{SN}, \mathrm{Pal}$ SK. Thyroid disorders in polycystic ovarian syndrome subjects: A tertiary hospital based crosssectional study from Eastern India. Indian J Endocr Metab. 2013;17:304-9.

2. Ramanand SJ, Ghongane BB, Ramanand JB, Patwardhan MH, Ghanghas RR, Jain SS. Clinical characteristics of polycystic ovary syndrome in Indian women. Indian J Endocr Metab. 2013;17:13845.

3. Rotterdam ESHRE/ASRM-Sponsored PCOS consensus workshop group. Revised 2003 consensus on diagnostic criteria and long-term health risks related to polycystic ovary syndrome. Fertil Steril. 2004;81:19-25.

4. Misra A, Chowbey P, Makkar BM, Vikram NK, Wasir JS, Chadha D, et al. Consensus statement for diagnosis of obesity, abdominal obesity and the metabolic syndrome for Asian Indians and recommendations for physical activity, medical and surgical management. J Assoc Physicians India. 2009;57:163-70.

5. Stein IF, Leventhal ML. Amenorrhea associated with bilateral polycystic ovaries. Am J Obstet Gynecol. 1935;29:181.

6. Knudsen N, Laurberg P, Rasmussen LB, Bülow I, Perrild H, Ovesen L, et al. Small differences in thyroid function may be important for body mass index and the occurrence of obesity in the population. J Clin Endocrinol Metab. 2005;90:401924.

7. Futterweit W, Dunaif A, Yeh HC, Kingsley P. The prevalence of hyperandrogenism in 109 consecutive female patients with diffuse alopecia. J Am Acad Dermatol. 1988;19:831-6.

8. Church RE. Hypothyroid hair loss. Br J Dermatol. 1956;77:661-3.

9. Sterry W, Konrads A, Nase J. Alopecia in thyroid diseases: Characteristic trichograms. Hautarzt. 1980;31:308-14.

10. Ober KP. Acanthosis nigricans and insulin resistance associated with hypothyroidism. Arch Dermatol. 1985;121:229-31.

11. Pfeifer SM, Kives S. Polycystic ovary syndrome in the adolescent. Obstet Gynecol Clin North Am. 2009;36(1):129-52.

12. Verma I, Sood R, Juneja S, Kaur S. Prevalence of hypothyroidism in infertile women and evaluation of response of treatment for hypothyroidism on infertility. Int J Appl Basic Med Res. 2012;2:17-9.

13. Tagawa N, Tamanaka J, Fujinami A, Kobayashi Y, Takano T, Fukata S, Kuma K, et al. Serum dehydroepiandrosterone, dehydroepiandrosterone sulfate, and pregnenolone sulfate concentrations in patients with hyperthyroidism and hypothyroidism. Clin Chem. 2000;46:523-8.

14. Casson PR, Lindsay MS, Pisarska MD, Carson SA, Buster JE. Dehydroepiandrosterone supplementation augments ovarian stimulation in poor responders: A case series. Hum Reprod. 2000;15:2129-32.

15. Dunaif A, Segal KR, Futterweit W, Dobrjansky A. Profound peripheral insulin resistance, independent of obesity, in polycystic ovary syndrome. Diabetes. 1989;38(9):1165-74.

16. Maratou E. Studies of insulin resistance in patients with clinical and subclinical hypothyroidism. Eur $\mathbf{J}$ Endocrinol. 2009;160:785-90.

17. Dittrich R, Kajaia N, Cupisti S, Hoffmann I, Beckmann MW, Mueller A. Association of thyroidstimulating hormone with insulin resistance and androgen parameters in women with PCOS. Reprod Biomed Online. 2009;19:319-25.

18. Isidro ML, Penín MA, Nemiña R, Cordido F. Metformin reduces thyrotropin levels in obese, diabetic women with primary hypothyroidism on thyroxine replacement therapy. Endocrine. 2007;32:79-82.

19. Gleicher N, Barad DH. Dehydroepiandrosterone (DHEA) supplementation in diminished ovarian reserve (DOR) Reprod Biol Endocrinol. 2011;9:67.

Cite this article as: Ramanand SJ, Raparti GT, Halasawadekar NR, Ramanand JB, Kumbhar AV, Shah RD. Hypothyroidism in polycystic ovarian syndrome: a comparative study of clinical characteristics, metabolic and hormonal parameters in euthyroid and hypothyroid polycystic ovarian syndrome women. Int J Reprod Contracept Obstet Gynecol 2016;5:3181-5. 